\title{
Gonococcal infection in Edinburgh and Newcastle: serovar prevalence in relation to clinical features and sexual orientation
}

\author{
J D C Ross, A Wardropper, M Sprott, A Moyes, H Young
}

\begin{abstract}
Aims-The variable distribution of gonococcal serovars in different areas is well recognised but the factors that are important determinants of serovar prevalence are less clear. The aim of this study was to identify relevant clinical variables by comparing serovar prevalence in two cities over the same time period.
\end{abstract}

Methods-A prospective analysis of serovar prevalence was made between January and December 1992 in Edinburgh and Newcastle with respect to age, sex, sexual orientation, antibiotic sensitivity and presence of symptoms.

Results -224 infective episodes of gonorrhoea were studied. The serovar distribution varied between the two cities with serovar 1B-1 being more common in Edinburgh (20/91 cf. 4/133, p < 0.01) and serovar 1B-6 more common in Newcastle (26/133 cf. 2/91, p < 0.01). Serovar 1A-2 was associated with heterosexual infection (35/114 in heterosexuals cf. 0/85 in homosexuals, $p<0.01$ ) and was more sensitive to penicillin than average (39/39 1A-2 strains highly penicillin sensitive cf. 98/184 for all other strains, $p<0.01$ ) whilst 1B-6 was mostly acquired through homosexual contact (22/26 cf. 63/142 for all other strains, $p<0.01$ ) and tended to show reduced penicillin susceptibility (13/28 1B-6 strains less penicillin sensitive cf. $45 / 195$ for all other strains, $p<$ 0.01). Infection with serovar 1A-2 was significantly less often symptomatic in heterosexuals than average (15/33 asymptomatic 1A-2 infections cf. $17 / 59$ for all other serovars, $p=0.015$ ). Subgroup analysis of male heterosexual infections confirms an association between asymptomatic infection and serovar 1A-2 (2/14 asymptomatic 1A-2 infections cf. $1 / 72$ for all other serovars, $p=0.02$ ). The distribution of infections over the year differed between the cities. Conclusions-A variety of factors including penicillin sensitivity and virulence may be important in determining the prevalence of gonococcal serovars within a given area.

(Genitourin Med 1994;70:35-39)

\section{Introduction}

The ability to serotype Neisseria gonorrhoeae using monoclonal antibodies has permitted detailed epidemiological studies of gonococcal infection over the past seven years. ${ }^{1-4} \mathrm{~A}$ varying distribution of serovars has been demonstrated both geographically ${ }^{56}$ and temporally..$^{78}$ Factors involved in producing geographical, temporal and sexual orientation associated differences in serovar prevalence are as yet unknown. Serovars isolated from groups within a population also differ with respect to serovar pattern. We have previously shown that serovars isolated from gay men in Edinburgh not only are different when compared with heterosexually acquired infections, but also that within the homosexual group dynamic change occurs in the prevalent serovar pattern. ${ }^{9}$ The association between clinical presentation and gonococcal serotype may help to provide the answer to this question. This prospective study was designed to observe and compare the patterns of gonococcal serotypes isolated in two cities in the United Kingdom with respect to their clinical features.

\section{Methods}

All patients presenting to the Departments of Genitourinary Medicine at Edinburgh Royal Infirmary or Newcastle General Hospital between January and December 1992 were analysed. The diagnosis of gonorrhoea was made on the basis of culture of $N$ gonorrhoeae on modified New York culture medium from the urethra, rectum, endocervix and/or throat. All male patients had a single urethral swab taken whilst female patients had urethral and endocervical swabs cultured on two separate occasions to diagnose or exclude gonorrhoea. Throat cultures were performed in all partners of patients with gonorrhoea and when the history indicated that this site had been placed at risk. Rectal cultures were taken routinely from men who gave a history of homosexual contact and in all women in Edinburgh. In Newcastle rectal cultures were taken when the history indicated that the site had been placed at risk, in those admitting to casual contacts and in contacts of patients known to be infected with gonorrhoea.

Gonococcal isolates were identified on the basis of biochemical and immunological tests and serotyping was performed using the American panel of monoclonal antibodies as has been described previously. ${ }^{10}$ Minimum inhibitory concentrations (MICs) of penicillin were determined by an agar plate dilution method ${ }^{5}$ using a series of plates incorporating $0.015,0.06,0.12,0.5$ and $1.0 \mathrm{mg} / 1$ of peni- 
cillin: MICs less than or equal to $0 \cdot 12 \mathrm{mg} / 1$ were classed as sensitive and isolates with MICs greater than or equal to 0.5 as reduced susceptibility.

Information was obtained from the casenotes of patients with a diagnosis of gon-

Table 1 Distribution of serovars in Edinburgh and Newcastle

\begin{tabular}{llcc}
\hline Serovar & Edinburgh & Newcastle & Total \\
\hline 1A-2 & 16 & 24 & 40 \\
1B-1 & $20 \dagger$ & 4 & 24 \\
1B-2 & 24 & 48 & 72 \\
1B-6 & 2 & $26 \dagger$ & 28 \\
1A-6 & 4 & 1 & 5 \\
1A-16 & 5 & 3 & 8 \\
1A-25 & 2 & 0 & 2 \\
1B-00` & 0 & 2 & 2 \\
1B-3 & 4 & 15 & 19 \\
1B-4 & 0 & 1 & 1 \\
1B-5 & 4 & 2 & 6 \\
1B-7 & 6 & 2 & 8 \\
1B-16 & 1 & 1 & 2 \\
1B-19 & 0 & 2 & 2 \\
1B-26 & 1 & 0 & 3 \\
1B-29 & 1 & 0 & 1 \\
Not Typed & 1 & 133 & 1 \\
Total & 91 & & 224 \\
\hline
\end{tabular}

${ }^{\star}$ untypable strains. tp $<0.01$.

Table 2 Comparison of Edinburgh and Newcastle cohorts

\begin{tabular}{llcc}
\hline Characteristic & & Edinburgh & Newcastle \\
\hline Age Group (years) & $15-19$ & 9 & 16 \\
& $20-24$ & 23 & 46 \\
& $25-29$ & 31 & 31 \\
Sex & $30-34$ & 11 & 18 \\
& over 34 & 15 & 14 \\
\multirow{4}{*}{ Sexual Orientation } & Heterosexual & 51 & 63 \\
& Male & 64 & 105 \\
\multirow{4}{*}{ Serogroup } & Homosexual & 31 & 54 \\
& Bisexual & 4 & 8 \\
& 1A & 27 & 28 \\
& 1B & 63 & 105 \\
\hline
\end{tabular}

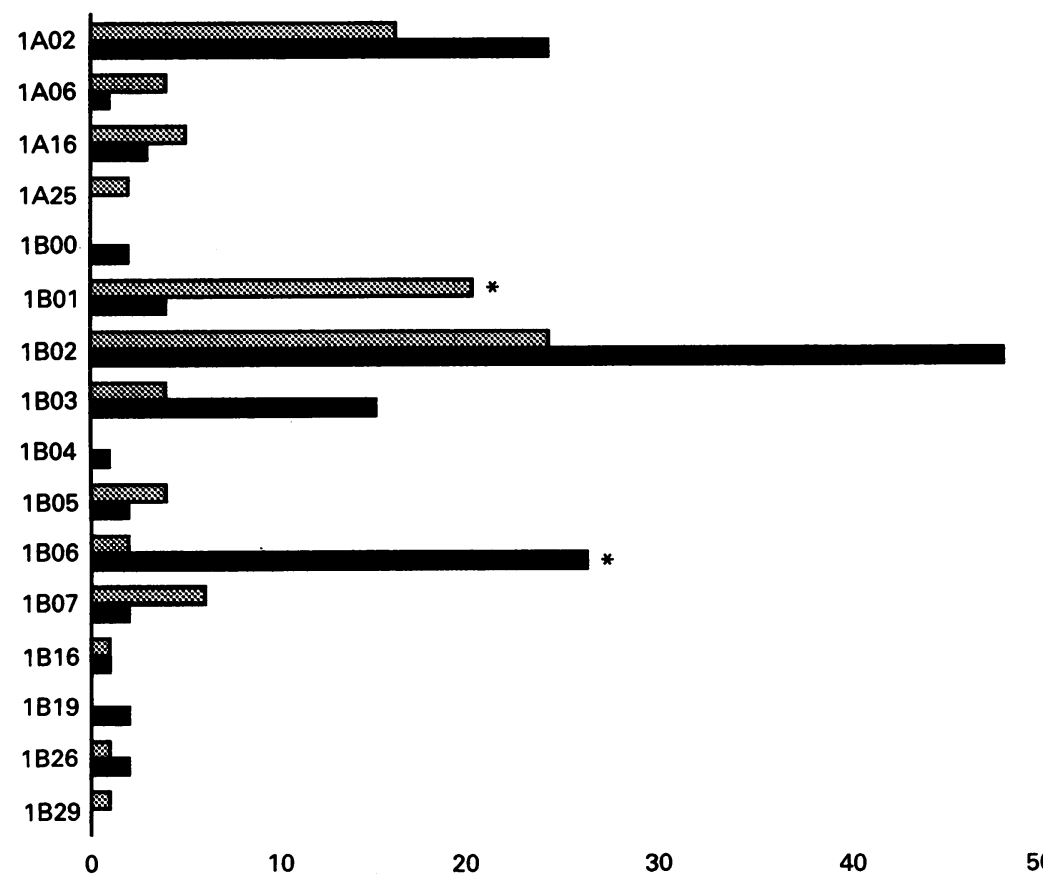

${ }^{*} p<0.01$

No of Infections

Edinburgh Newcastle

Figure 1 Frequency of gonococcal serovars in Edinburgh and Newcastle. orrhoea on sex, sexual orientation, antibiotic sensitivity, date of diagnosis and presence of symptoms. The data on serovar prevalence in Edinburgh and Newcastle were then analysed with respect to the clinical information. The data were entered into the DBase (Borland $\mathcal{D}_{\overparen{D}}$ Software) database programme and statistical analysis was performed using chi-square on the Epiinfo statistical package (WHO public domain software).

\section{Results}

In Edinburgh, 91 episodes of gonorrhoea in 91 patients were analysed. Gonococcal serovar data was available in 90 (99\%) cases, $\overparen{\Phi}$ sexual orientation in $86(95 \%)$, antibiotic sensitivity in $91(100 \%)$, date of diagnosis in $91(100 \%)$ and details of symptoms in $85^{\circ}$ (93\%). Newcastle had 133 episodes of gonor- $\vec{\omega}$ rhoea in 126 patients. Serovar data was available in 133 cases $(100 \%)$, sexual orientation = in $125(94 \%)$, antibiotic sensitivity in 132 . $(99 \%)$, date of diagnosis in $133(100 \%)$ and details of symptoms in 120 (90\%)

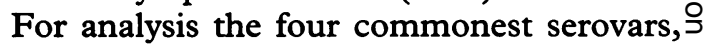
which accounted for $73 \%$ of infections (1A-2, $\overrightarrow{-}$ 1B-1, 1B-2 and 1B-6), were looked at separately whilst the other "minor" serovars were grouped together. Serovar incidence is shown in table 1.

No significant differences were seen in the age $(p=0.24)$, sex ratio $(p=0.24)$, sexual orientation $(p=0.43)$ or ratio of $1 \mathrm{~A}$ to $1 \mathrm{~B}$ infections $(p=0.17)$ in infections in Edinburgh compared with Newcastle (table⿳亠丷厂

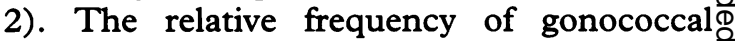
serovars in the two cities is shown in fig $1 . \overrightarrow{\vec{F}}$ Serotype 1B-1 infection was significantly more common in Edinburgh while $1 \mathrm{~B}-6$ ? infection was seen more frequently in? Newcastle over the 12 month study periodon (table 1). The distribution of infections through the year differed between Edinburgh and Newcastle with the peak number ofo infections occurring in the 2 nd quarter in Newcastle ( $p=0.05$ ) (fig 2).

Serovar 1A-2 was associated exclusively $\frac{\text { I }}{2}$ with heterosexual infection (fig 3). 1B-2 infections were significantly associated with

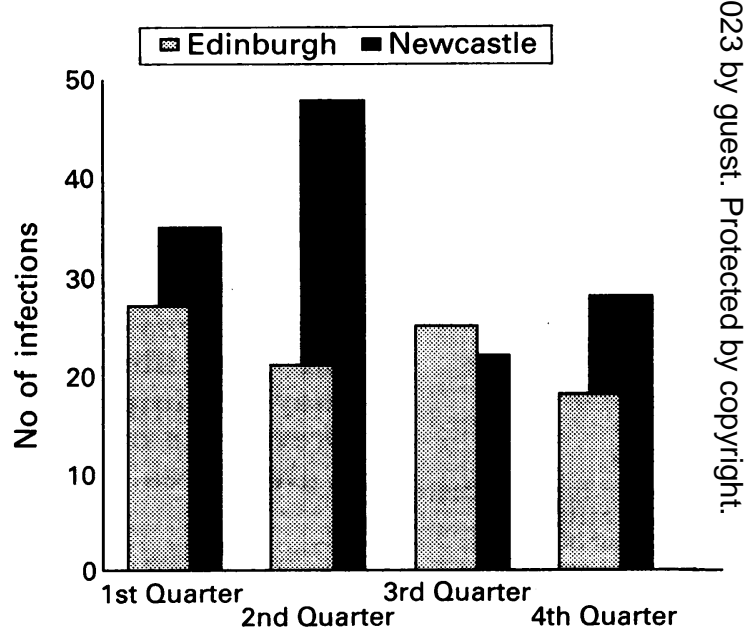

Figure 2. Quarterly frequencies of gonococcal infection in Edinburgh and Newcastle. 


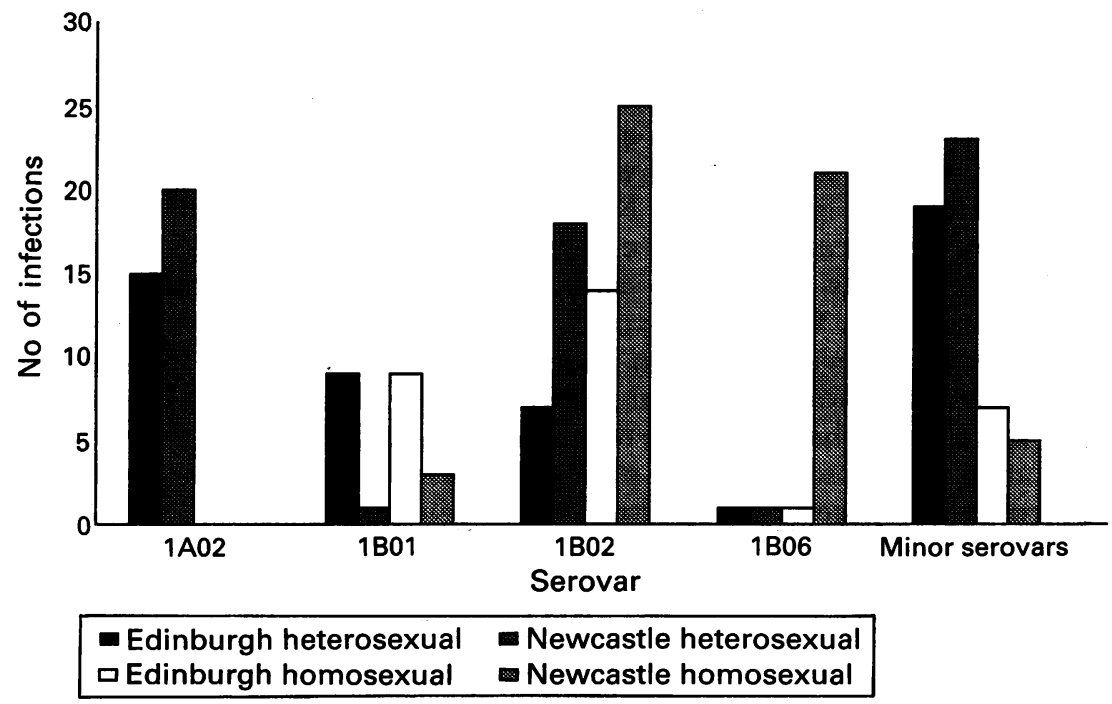

Figure 3 Frequency of gonococcal serovars in Edinburgh and Newcastle subdivided by sexual orientation. showed an association between asymptomatic infection and serovar 1A-2 (2/14 asymptomatic male genital infection for serovar $1 \mathrm{~A}-2$ cf. 1/72 for all other serovars, $p=0.02$ ). The age distribution of infected patients did not differ for individual serovars.

\section{Discussion}

The observed pattern of gonococcal serovars varies between Edinburgh and Newcastle although geographically the cities are not far apart. Certain serovars, such as 1A-2 and 1B2 , are observed in both cities whilst others predominate in one area only: $1 \mathrm{~B}-6$ in Newcastle and 1B-1 in Edinburgh, despite the patients being similar with respect to age, sex and sexual orientation. As has been previously reported some serovars are associated with certain sexual behaviour patterns ${ }^{17}$ and we found $1 \mathrm{~A}-2$ to be exclusively heterosexually acquired while 1B- 6 was usually acquired through homosexual contact, although 1B-2 was found in substantial numbers of both homosexual and heterosexual patients. Although serovar 1B-6 was isolated only very infrequently in Edinburgh in 1992, compared with Newcastle, previous reports have showed that this serovar was prevalent in gay men in Edinburgh in 1991 and was also increasing in prevalence in Glasgow during the same time period. ${ }^{5}$ It has been postulated that serovars may "cross over" from homosexual to heterosexual populations or vice versa via bisexual men. It is also possible that certain gonococcal strains have a selective advantage in gay men by, for example, being able to thrive more successfully in the rectal environment. ${ }^{11}$

Plummer et $a l^{612}$ and Buchanan et al ${ }^{13}$ have suggested that there may be a serovar specific immune response which gives partial protection against reinfection with the same gonococcal strain. This implies that the success of a "new" strain within any particular community would depend on that community's previous exposure and may explain the differences observed in this and other studies which have compared serovar patterns in different geographical areas of even relatively close proximity. ${ }^{5}$

Over the past decade the incidence of gonorrhoea has fallen dramatically in many parts of the Western world ${ }^{14}$ although there has recently been an increase reported in England and Wales. ${ }^{15}$ No such increase has thus far occurred in Edinburgh, with the exception of an unsustained rise in homosexually acquired gonorrhoea, ${ }^{16}$ but the observed decline in the incidence of gonorrhoea has plateaued (unpublished data). Widespread media campaigns directed at preventing HIV infection over the same time period would therefore appear to have affected sexual behaviour as measured by the incidence of other sexually acquired infections although this effect is of short duration. ${ }^{17}$ If serovar characteristics themselves were important in determining their prevalence within a population then selective pressure on gonococcal strains 
may be expected to increase as the overall incidence of gonorrhoea declines with the emergence of a few successful serovars. We have however, observed a comparatively large number of different serovars with relative success occurring in only a few. This implies that either there is a continual influx of unsuccessful strains from other geographical areas or else population factors are also important. We have previously shown that certain serovars can persist at low levels within a population whilst others appear only transiently ${ }^{9}$ which lends support to the importance of population -behaviour in a serovar's success. Homosexual populations often have infections with serovars that are uncommon in the heterosexual population ${ }^{9}$ and this is likely to be due, at least partially, to relatively infrequent sexual mixing via bisexual men.

The presence of different serotypes of $N$ gonorrhoeae is presumably as a result of the antigenic heterogeneity which occurs secondary to genetic mutation and indeed a number of "evolutionary trees" have been propösed. ${ }^{18}$ The relevance of this "antigenic drift" to changing serovar patterns over time is not known as the rate of mutation in vivo has never been assessed. One way of estimating this is by comparing serovar patterns in named -sexual-contacts although this has given conflicting results with some authors finding a high correlation between partners ${ }^{19}$ and others less so. ${ }^{20}$

The quarterly frequencies of gonococcal isolation in Edinburgh, peaking in the 1st and 3rd quarters, were the reverse of that observed in Newcastle. The pattern seen in Edinburgh follows that reported in the whole of Scotland in the past ${ }^{21}$ which was postulated to be secondary to the increased mobility of the young sexually active population during holiday periods.

The absence of symptoms would in theory be advantageous to a serovar in natural selection by increasing the chance of more contacts being infected prior to detection and treatment. Serovar 1A-2 produces less symptoms than average in heterosexually acquired genital infections in men and is also one of the most successful strains in both Edinburgh and Newcastle."- Although Crawford et al ${ }^{22}$ found that-the AHU auxotype of gonorrhoea was less likely to produce symptoms a recent article by Horner et al ${ }^{23}$ found no difference in the symptoms caused by $1 \mathrm{~A}$ and $1 \mathrm{~B}$ serogroups. The possible explanations for the disparity between our results and those of Horner may relate to either the different populations studied or methodological differences. Serotype 1A-2 was the predominant 1A serovar in our population and if lack of symptoms were related to this particular serovar rather than serogroup $1 \mathrm{~A}$ in general this may bias the serogroup analysis in areas where $1 \mathrm{~A}-2$ is less prevalent. There are also a number of potential flaws in the analysis of Horner's results. The number of patients included for most of their analysis is small (55) and $15 \%$ of the patients who completed questionnaires were not serogrouped. The analysis of presence or absence of symptoms was based on 91 patients in 68 of whom the information appeared to have been obtained from questionnaire while in 36 the notes were used which makes valid interpretation difficult. No comparison between data obtained from case notes and questionnaire was made. The association between symptoms and serotype is further complicated by the observation that symptoms associated with gonococcal infection may not necessarily remain static over time and an increase in the incubation period from 1932 to 1989 has been reported. ${ }^{24}$

In common with other studies we found serogroup 1A more sensitive to penicillin than serogroup 1B. ${ }^{25-30}$ In particular serovar 1A-2 was universally sensitive although others have demonstrated that this strain can become chromosomally resistant with one study showing $6 / 278(2 \cdot 2 \%)$ of $1 \mathrm{~A}-2$ infections to be CMRNG. ${ }^{31}$ Despite this sensitivity to antibiotics, infections with $1 \mathrm{~A}-2$ are very successful in the community indicating that there may be some selective advantage associated with this trait.

The results of this and other studies indicate a complex interaction between the characteristics of the individual, the population and the gonococcal strain itself in determining the prevalence of a particular serovar in the community. Further research at both the epidemiological and molecular levels will be required before these issues can be resolved.

1 Young H, Moyes A, Ross JDC, McMillan A. A serovar analysis of heterosexual gonorrhoea in Edinburgh analysis of heterosexual gonorrhoea

2 Bygdeman S. Polyclonal and monoclonal antibodies applied to the epidemiology of gonococcal infections. In: Young $\mathrm{H}$, McMillan A, eds. Immunological Diagnosis of Sexually Transmitted Diseases. New York: Mark Dekker, 1987:117-65.

3 Knapp JS, Sandstrom E, Holmes KK. Overview of epidemiological and clinical applications of auxotype/ serovar classification of Neisseria gonorrhoeae. In: Schoolnik GK, ed. The Pathogenic Neisseriae. Washington D.C.: American Society for Microbiology, 1985:6-12.

4 Kohl PK, Knapp JS, Hofmann H, et al. Epidemiological analysis of Neisseria gonorrhoeae in the Federal Republic of Germany by auxotyping and serological Republic of Germany by auxotyping and serological
classification using monoclonal antibodies. Genitourin Med 1986;62:145-50.

5 Young H, Moyes A, Robertson DHH, et al. Gonococcal infection within Scotland: antigenic heterogeneity and antibiotic susceptibility of infecting strains. Eur $\mathcal{f}$ Epidemiol 1990;6:1-8.

6 Plummer FA, Brunham RC. Gonococcal recidivism, diversity, and ecology. Rev Infect Dis 1987;9:846-50.

7 Knapp JS, Holmes KK, Bonin P, Hook EW. Epidemiology of gonorrhoea: distribution and temporal changes in auxotype/serovar classes of Neisseria gonorrhoeae. Sex Transm Dis 1987;14:26-32.

8 Gill MJ. Serotyping Neisseria gonorrhoeae: a report of the Fourth International Workshop. Genitourin Med 1991;67:53-7.

9 Young H, Moyes A, Ross JDC, McMillan A. Patterns of homosexually acquired gonococcal serovars in Edinburgh 1986-90. Genitourin Med 1991;67:312-6.

10 Moyes A, Young H. Epidemiological typing of Neisseria gonorrhoeae: a comparative analysis of three monoclonal antibody serotyping panels. Eur $\mathfrak{f}$ Epidemiol 1991;7:311-9.

11 Morse SA, Lysko PG, McFarland L, et al. Gonococcal strains from homosexual men have outer membranes with reduced permeability to hydrophobic molecules. Infect Immun 1982;37:432-8.

12 Plummer FA, Simonsen JN, Chubb $\mathrm{H}$, et al. Epidemiologic evidence for the development of serovarspecific immunity after gonococcal infection. $f \mathrm{Clin}$ Invest 1989;83:1472-6. 
13 Buchanan TM, Eschenbach DA, Knapp JS, Holmes KK Gonococcal salpingitis is less likely to recur with
Neisseria gonorhoeae of the same principal outer memNeisseria gonorhoeae of the same principal outer mem-
brane antigenic type. Am $f$ Obstet Gynecol 1980;138: 978-80.

14 Donovan B, Bek MD, Pethebridge AM, Nelson MJ Heterosexual gonorrhoea in central Sydney: implications for HIV control. Med Y Aust 1991;154:175-80.

15 Evans BA, Catchpole MA, Heptonstall J. Sexually transmitted diseases and HIV-1 infection among homosexual men in England and Wales. BMF 1993;306:426-8.

16 Ross JDC, McMillan A, Young $\mathrm{H}$. Changing trends of gonococcal infection in homosexual men in Edinburgh. Epidemiol Infect 1991;107:585-90.

17 Ross JDC, Scott GR. The association between HIV media campaigns and number of patients coming forward for HIV antibody testing. Genitourin Med 1993;69:193-5.

18 Sandstrom E, Tam M, Bygdeman S. Antigenic drift of gonococcal protein I as judged by serovar determination. In: Schoolnik GK, ed. The Pathogenic Neisseriae. Washington D.C.: American Society for Microbiology, 1985:13-9.

19 Ison CA, Whitaker L, Renton A. Concordance of auxotype/serovar classes of Neisseria gonorrhoeae between sexual contacts. Epidemiol Infect 1992;109:265-71.

20 Young H, Moyes A, Ross JDC, McMillan A, Robertson DH. Serotype patterns of gonococcal infection in conDH. Serotype patterns of gonococcal in

21 Ross JDC, Scott GR. Seasonal variation in gonorrhoea. Eur $₹$ Epidemiol 1992;8:252-8.

22 Crawford G, Knapp JS, Hale J, Holmes KK Asymptomatic gonorrhoea in men: caused by gonococci with unique nutritional requirements. Science 1977;196:
1352-3.

23 Horner PJ, Coker RJ, Turner A, Shafi MS, Murphy SM Gonorrhoea: signs, symptoms and serogroups. Int $\mathscr{f}$ STD AIDS 1992;3:430-3.

24 Sherrard J, Barlow D. Gonorrhoea in men [letter]. Lance 1993;341:245.

25 Brett MS, Davies HG, Blockley JR, Heffernan HM Antibiotic susceptibilities, serotypes and auxotypes of Neisseria gonorrhoeae isolated in New Zealand. Genitourin Med 1992;68:321-4.

26 Woodford N, Bindayna KM, Easmon CS, Ison CA. Associations between serotype and susceptibility to antibiotics of Neisseria gonorrhoeae. Genitourin Med 1989;65:86-91.

27 Abeck D, Johnson AP, Korting HC. Characterisation of penicillinase producing gonococci isolated in Munich, 1981-6. Genitourin Med 1988;64:3-6.

28 Danielsson D, Bygdeman S, Kallings I. Epidemiology of gonorrhoea: serogroup, antibiotic susceptibility and auxotype patterns of consecutive gonococcal isolates from ten different areas of Sweden. Scandi $\mathcal{F}$ Infect Dis $1983 ; 15: 33-42$.

29 Dillon JR, Bygdeman SM, Sandstrom EG. Serological ecology of Neisseria gonorrhoeae (PPNG and nonPPNG) strains: Canadian perspective. Genitourin Med 1987;63:160-8.

30 Rice RJ, Biddle JW, JeanLouis YA, DeWitt WE, Bloun JH, Morse SA. Chromosomally mediated resistance in Neisseria gonorrhoeae in the United States: results of surveillance and reporting, 1983-1984. I Infect Dis 1986;153:340-5.

31 Ison CA, Easmon CS. Epidemiology of penicillin resistan Neisseria gonorrhoeae. Genitourin Med 1991;67:307-11. 